\title{
Experimental study on human target localization system using a multistatic ultra-wideband sensor
}

\author{
Ryohei Nakamura ${ }^{\text {a) }}$, Nana Shimizu, and Hisaya Hadama \\ Graduate School of Science and Engineering, National Defense Academy of Japan, \\ Hashirimizu, Yokosuka, Kanagawa 239-8686, Japan
}

a)nakamura@nda.ac.jp

\begin{abstract}
We have previously proposed a human target localization method using an ultra-wideband sensor with multistatic placed antennas and investigated its fundamental localization performance by computer simulation using simple indoor and human body models. In actual uses for the sensor, there are many reflectors such as desks and chairs other than walls in the room. Also, the intensity of the reflected wave from an actual human body changes complicatedly because the radar cross section varies greatly depending on the incident angle of radio waves. Therefore, in order to investigate the effectiveness of the proposed method, it is important to conduct experiments in actual radio propagation environment. In this research, we construct an experimental system that implements the proposed method and experimentally investigate the effectiveness of our proposal by changing the sensor parameters. The experimental results have confirmed that, for example, when the bandwidth is $3 \mathrm{GHz}$, the localization error was within $0.25 \mathrm{~m}$ with a probability of $80 \%$ or more, and the locations of a human target can be estimated with high accuracy.
\end{abstract}

Keywords: ultra-wideband, sensor, human target monitoring, multistatic, localization, experiment

Classification: Sensing

\section{References}

[1] M. Otsu, R. Nakamura, and A. Kajiwara, "Elderly-care monitoring sensor using stepped-FM UWB scheme," Proc. of 2012 IEEE Sensor Application Symposium (SAS2012), Brescia, Italy, pp. 151-154, Feb. 2012. DOI:10.1109/ SAS.2012.6166286

[2] R. Nakamura and A. Kajiwara, "Development of indoor intruder detection sensor system using ultra-wideband stepped-FM and its verification," IEICE Trans. Commun., vol. J100-B, no. 12, pp. 1033-1038, Dec. 2017 (in Japanese). DOI:10.14923/transcomj.2017ASL0001

[3] R. Nakamura, M. Kurosaki, and H. Hadama, "Localization performance of multi-static ultra-wideband radio sensor for indoor human monitoring system," IEICE Trans. Commun., vol. J100-B, no. 12, pp. 974-983, Dec. 2017 (in Japanese). DOI:10.14923/transcomj.2017ASP0001 
[4] M. I. Skolnik, Radar Handbook: Third Edition, McGraw-Hill, 2008.

[5] I. Matsunami, R. Nakamura, and A. Kajiwara, "RCS measurements for vehicles and pedestrian at 26 and $79 \mathrm{GHz}$," IEICE Trans. Fundamentals, vol. E99-A, no. 1, pp. 204-206, Jan. 2016. DOI:10.1587/transfun.E99.A.204

[6] E. D. Kaplan and C. J. Hegarty, Understanding GPS: Principles and Applications Second Edition, Artech House, 2006.

[7] K. Atkinson, An Introduction to Numerical Analysis: Second Edition, John Wiley \& Sons, 1989.

\section{Introduction}

Recently, the interest in human monitoring systems that detect invasion crimes or fall accidents of elderly persons is rising. For these systems, the use of ultrawideband (UWB) radio sensors has investigated and various researches have been reported [1, 2, 3]. Particularly, in [3], we have proposed a human target localization method using an UWB sensor with multistatic placed antennas and investigated its fundamental localization performance by computer simulation. The method, only to place two or more omni-directional receiving antennas with a conventional monostatic placed antenna, can estimate location of the target in addition to distance of the target. Since the proposed method is a system that adds a plurality of omnidirectional receiving antennas without changing the conventional antenna, it is possible to apply the conventional monitoring functions as it is. In the computer simulation, we investigated the effect of sensor parameters on localization performance in a radio propagation environment composed of an indoor model without furniture and a simple human body model simulated by a quadratic prism using a ray tracing simulator. However, in actual uses, there are many reflectors such as desks and chairs other than walls in the room. Also, the intensity of the reflected wave from an actual human body changes complicatedly because the radar cross section (RCS) varies greatly depending on the incident angle of radio waves $[4,5]$. Therefore, in order to investigate the effectiveness of the proposed method, it is important to conduct experiments in actual radio propagation environment.

In this letter, we experimentally investigate the effectiveness of our proposal by constructing an experimental system that implements the human target localization algorithm using a multistatic UWB sensor.

\section{Target localization method using a multistatic UWB sensor}

The UWB sensor measures received signal intensity as a function of distance called "range profile". Suppose actual indoor sensing situations, the profile includes multiple paths reflected from walls and furniture as well as a target. Therefore, existence of a target in a room can be estimated from changes in range profiles [2]. In particular, the existence is detected by using "difference range profile" which is expressed by

$$
\Delta R^{i}(d)=\left|R^{i}(d)-R^{0}(d)\right|
$$




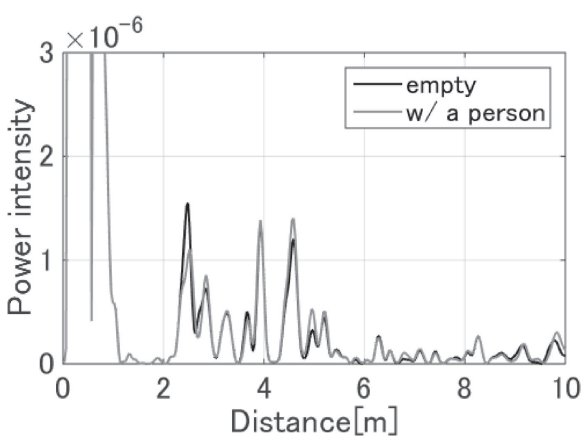

(a) an example of range profiles

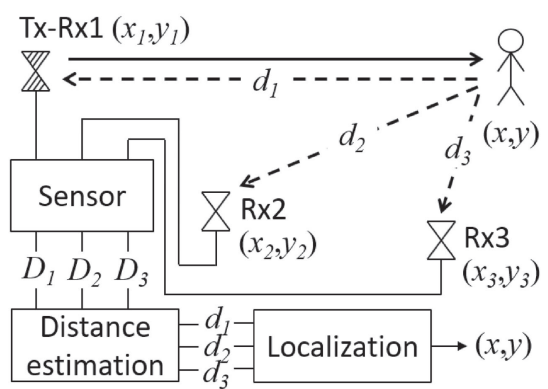

(b) concept of localization method

Fig. 1. Target localization method using a multistatic UWB sensor.

movement and distance can be estimated to compare $R^{0}(d)$ with $R^{i}(d)$. Fig. 1(a) shows an example of experimentally measured range profiles with and without a human target in a room, where the bandwidth is $1 \mathrm{GHz}$ and the target is standing at a distance of $2.5 \mathrm{~m}$ from a monostatic-placed antenna. The large changes in power intensity are seen at a distance of $2.5 \mathrm{~m}$ or more. This means that many multipaths are blocked or reflected by the human body. Thus, by detecting the distance at which the power difference exceeds any threshold for the first time, the distance of the target can be estimated.

Localization systems such as GPS generally estimate distances from observation points of known coordinates to an unknown target and perform localization of the unknown target by solving nonlinear simultaneous equations based on the known coordinates and the estimated distances [6]. If the sensor can estimate the distances between observation points of known coordinates and a human target, coordinates of the target can be estimated. Thus, we have considered to use a plurality of receiving antennas placed at different places with known coordinates [3]. Fig. 1(b) illustrates a concept of the proposed localization method, where $(x, y)$ is unknown coordinates of the target, $\left(x_{1 \sim 3}, y_{1 \sim 3}\right)$ are known coordinates of the receiving antennas. In this method, two or more omni-directional receiving antennas $(\mathrm{Rx} 2 / \mathrm{Rx} 3)$ are used in addition to the conventional monostatic placed transceiving antenna (Tx-Rx1), that is, a multistatic sensor. In Fig. 1(b), $D_{1} \sim D_{3}$ are estimated distance information for each of the Tx-Rx\# antenna sets. It is noted that the information is expressed in bi-static range, which means the radio propagation distance between transmitting and receiving antennas. Thus, the distances from the Rx antennas to the target $d_{1} \sim d_{3}$ are estimated by following equations.

$$
d_{1}=D_{1} / 2, \quad d_{2}=D_{2}-d_{1}, \quad d_{3}=D_{3}-d_{1}
$$

Subsequently, the target's unknown coordinates $(x, y)$ are estimated by solving nonlinear simultaneous equations based on the estimated distances $d_{1} \sim d_{3}$ and the known coordinates of the receiving antennas $\left(x_{1 \sim 3}, y_{1 \sim 3}\right)$ as with the general localization systems. In this paper, Newton-Raphson method [7] is used for finding the numerical solution of the simultaneous equations.

\section{Experimental investigations on the proposed method}

In this research, we measured the range profiles of each transmitting and receiving antenna set using a vector network analyzer and constructed an experimental 


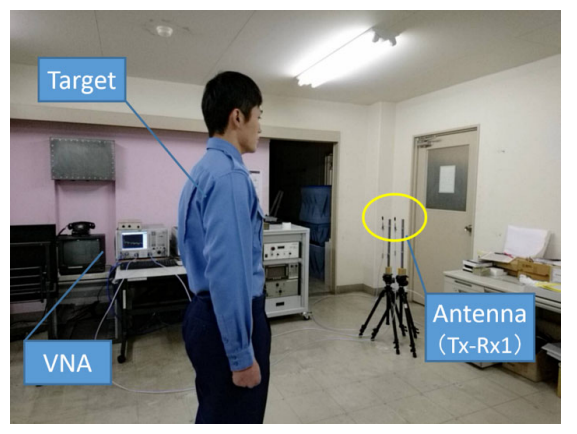

(a) measurement scene

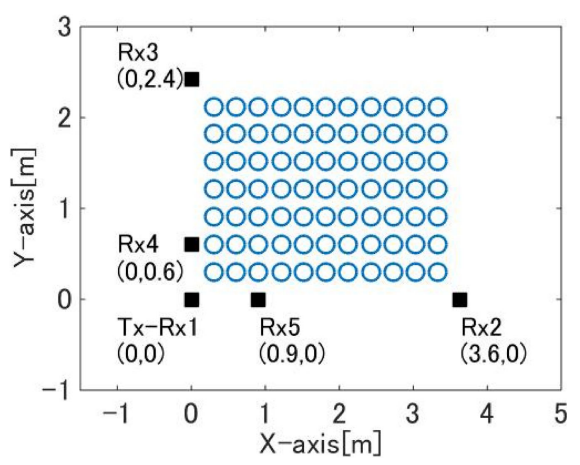

(b) target locations and antenna arrangement

Fig. 2. Measurement environment.

system that performs localization by the proposed method offline. The experiment was conducted in the indoor environment $(4 \mathrm{~m} \times 5 \mathrm{~m})$ as shown in Fig. 2(a). The human target (height: $1.81 \mathrm{~m}$ ) was standing without moving in order at the locations indicated by circles in Fig. 2(b) (total 77 points at $0.3 \mathrm{~m}$ intervals). Also, transmitting and receiving antennas Tx-Rx1 and receiving antennas $\mathrm{Rx} 2, \mathrm{Rx} 3, \mathrm{Rx} 4$, and $\mathrm{Rx} 5$ were arranged at the locations indicated by squares in Fig. 2(b). For each of the antennas, an omni-directional antenna $(2.6 \mathrm{dBi})$ was used and arranged at a height of $1.2 \mathrm{~m}$ above the floor. In the measurements, the center frequency was set to $4.5 \mathrm{GHz}$ and the bandwidth $B W$ was set to $1 \mathrm{GHz}$ and $3 \mathrm{GHz}$. We took localization analyses for the case in which the antennas Tx-Rx1, Rx2, and $\mathrm{Rx} 3$ were selected (arr. 1), and the case in which the antennas Tx-Rx1, Rx4, and $\mathrm{Rx} 5$ were selected (arr. 2).

Figs. 3(a) and 3(b) show the localization results at each of the 77 points for $B W=1 \mathrm{GHz}$ and $B W=3 \mathrm{GHz}$ when the antenna arrangement is arr. 1, respectively. In these figures, the circles and crosses indicate actual and estimated locations of the human target, respectively. From Fig. 3(a), it is seen that the target locations can be estimated with relatively high accuracy. However, it is also seen that the localization errors exceeding $1 \mathrm{~m}$ occur at a few measurement points. This is because the RCS of human body greatly varies depending on the incident angle of radio waves, the sensitivity is lowered, and the ranging error for each antenna set is increased. Since the decrease in sensitivity is temporary, the correlation with the errors of the adjacent measurement points is small. Moreover, these figures confirm that the localization accuracy for $B W=3 \mathrm{GHz}$ is improved as compared with that for $B W=1 \mathrm{GHz}$. This is because the range resolution is improved by using a wider band.

In order to quantitatively evaluate the localization performance, Figs. 3(c) and 3(d) show cumulative distribution functions (CDF) of the localization errors for all the measurement points in each of the antenna arrangements. From Fig. 3(c), we can see that our proposed method provides good estimates of the target's locations. For example, in the case of $B W=1 \mathrm{GHz}, 80 \%$ of the localization errors are $0.3 \mathrm{~m}$ or less. Furthermore, in the case of $B W=3 \mathrm{GHz}, 80 \%$ or more of the localization errors are $0.25 \mathrm{~m}$ or less, and all the errors are approximately $1 \mathrm{~m}$ or less. As can be seen in Fig. 3(d), the localization performance of antenna arrangement of arr. 2 tends to deteriorate compared to that of arr. 1. Specifically, the proportion of 


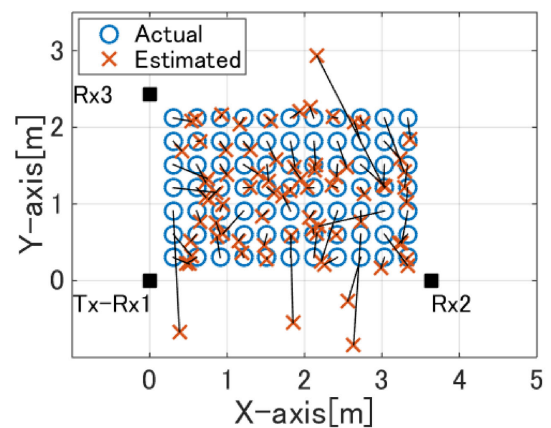

(a) localization results $(B W=1 \mathrm{GHz})$

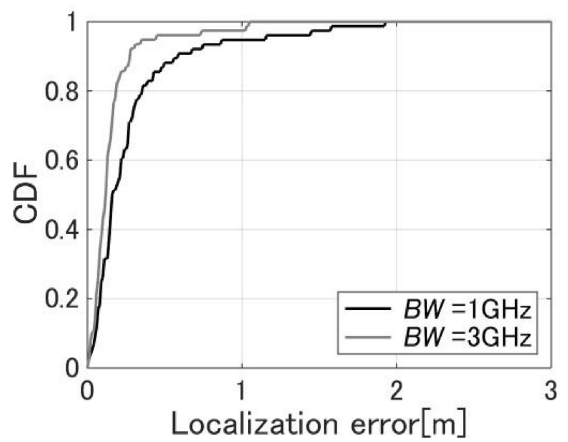

(c) CDF of localization errors (arr. 1)

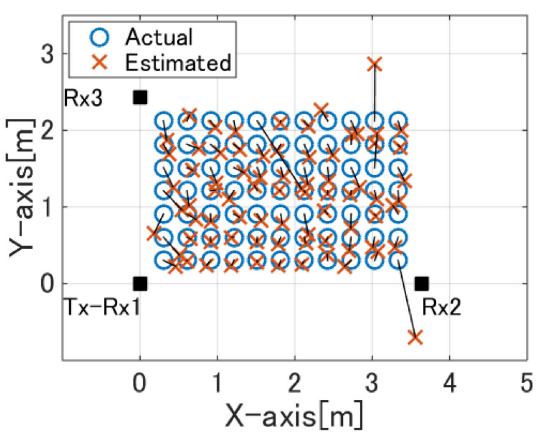

(b) localization results $(B W=3 \mathrm{GHz})$

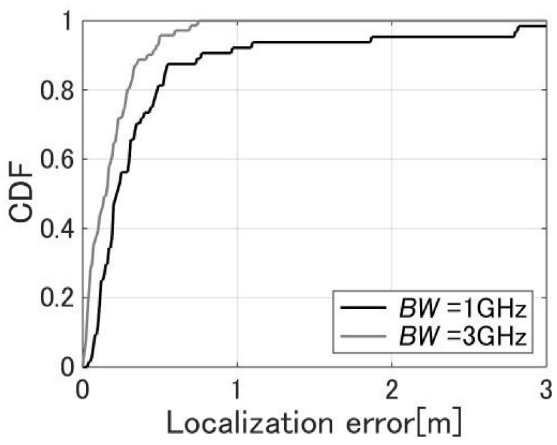

(d) CDF of localization errors (arr. 2)

Fig. 3. Experimental results.

measurement points with large errors increases. This is because an influence of the ranging accuracy on the localization performance increases due to narrowing of arrangement intervals of the antennas. However, even if arrangement intervals are small, we can mitigate the influence by increasing the bandwidth (improving the ranging accuracy). Unlike this experiment, in the simulation study reported by the authors in [3], the influence of bandwidth on the localization performance was small. This is because the sensitivity of the sensor was relatively high by simulating the indoor environment and the human body with simple models. In actual environments, the sensitivity to a human body considerably changes according to the incident angle of radio waves, so it is expected that the sensitivity sometimes decreases. Therefore, in actual use, we can conclude that increasing the bandwidth is effective.

\section{Conclusions}

In this research, we have constructed an experimental system that implements the human target localization method with a multistatic UWB sensor proposed in [3] and experimentally investigated the effectiveness of the proposed method. The experimental results confirmed that $80 \%$ or more of the localization errors are $0.3 \mathrm{~m}$ or less and $0.25 \mathrm{~m}$ or less when the bandwidth is $1 \mathrm{GHz}$ and $3 \mathrm{GHz}$, respectively, and the locations of a human target can be estimated with high accuracy. Even the human monitoring systems such as those proposed in [1] and [2] have a ranging error of approximately $0.3 \mathrm{~m}$. Therefore, the method is probably effective for these systems. Moreover, although the localization accuracy deteriorates by bringing the antennas close to each other, we also confirmed that the influence can be mitigated by increasing the bandwidth. 
In the future research, we will investigate the localization performance in NLOS environments. In addition, although the experimental system constructed in this research was a configuration that performs localization estimation offline, we will construct an experimental system that estimates the locations in real time. 